\title{
Paper
}

\section{MIMO Antenna for Ka Band and 5G Communication Applications}

\author{
B. T. P. Madhav, G. Jyothsna Devi, P. Lakshman, and T. Anilkumar \\ Department of Electronics and Communication Engineering, Koneru Lakshmaiah Education Foundation, Andhra Pradesh, India
}

https://doi.org/10.26636/jtit.2018.123717

\begin{abstract}
This article presents a MIMO compact antenna measuring $45 \times 45 \times 1.6 \mathrm{~mm}$, on the FR4 substrate, proposed for Ka band and $5 \mathrm{G}$ communication applications. The proposed design is suitable to overcome the issues connected with massive MIMO. It has four-sigma-shaped radiating elements and a c-shaped ground plane with coplanar waveguide feeding. Its compact dimensions suit it for most existing communications systems. The aerial operates in the $21-30 \mathbf{G H z}$ range, which covers $\mathrm{Ka}$ and $5 \mathrm{G}$ communication bands. The proposed antenna exhibits the average efficiency of more than $76 \%$ within its operating band and gives a minimum signal to noise plus interference ratio. The presented antenna covers several services, such as Ka band satellite downlink applications and future 5G communication applications.
\end{abstract}

Keywords-5G, coplanar waveguide, Ka band, MIMO, satellite downlink.

\section{Introduction}

The unsatisfied and unresolved requirements of 4G technology gave birth to the new 5G standard. In future mobile communications, $5 \mathrm{G}$ is going to offer solutions to common problems, i.e. poor coverage, crowded channels, poor quality, dropped connections, etc. To achieve flexibility in communication and to fulfil the users' expectations, there is a need to move forward with $5 \mathrm{G}$ even further. A 5G network not only provides high speed, but also offers the capacity to carry more data with data rates of over $1 \mathrm{Gbps}$ [1], [2]. Such a big capacity is going to make significant difference in communications and allows to expand the range of services offered (Fig. 1) [3], [4]. Low latency with massive connectivity and large system capacity are needed for the variety of novel applications of $5 \mathrm{G}$ systems [5]. 5G has the latency time on the millisecond level, and super-dense collections will improve spectral, energy as well as cost efficiency. Novel multiple access strategies, ultra-dense networking, all spectrum access, MIMO technology, full/flexible duplex and many other solutions are still under development [6]. 5G must support multiple novel applications with different requirements. The requirements include higher peaks, reduced latency, high user data rates, increased number of devices, enhanced indoor coverage and so on. This technology will provide coverage for high-edge users [7].

The increasing demand for high speed data, together with rising congestion within the microwave spectrum are pushing for higher frequencies, including $\mathrm{Ka}, \mathrm{Q}$ and $\mathrm{V}$ bands. The operating frequency of the Ka band is nearly 3 times higher than that of the $\mathrm{X}$ band [8]. This is because enhancement in satellite communications (SAT) is mainly concerned with high versatility, compactness, performance, low cost as well as low weight [9].

A fixed satellite service for wireless multimedia and internet access has generated renewed interest in the Ka band, using downlink frequencies of $17.72-21.2 \mathrm{GHz}$ and uplink frequencies of $27.52-30 \mathrm{GHz}$ in order to properly accommodate various future communications systems [10]. Satellite communication for the military (MKa band) offers an even wider bandwidth for high speed Internet access (30 GHz for TX and $20 \mathrm{GHz}$ for RX) [11]. Satellite communication plays a major role in offering access to the Internet in rural areas. To remove high path losses from LEO and GEO frequency satellites, high-gain antennas, i.e. dish antennas of the horn type are needed [12].

\section{Related Work}

To achieve a high data rate and to improve signal quality and reliability, MIMO antennas are considered [13], [14]. A MIMO antenna having 2 elements for a $5 \mathrm{G}$ network is mentioned in [15], [16]. The Ka band single polarization MIMO satellite-earth link channel model is shown in papers [17] and [18]. A novel single layer substrate integrated waveguide corrugated technique for two high gain and low mutual coupling Ka band MIMO antennas are demonstrated in [19].

\section{Massive MIMO Challenges}

Massive MIMO has several advantages over conventional MIMO antennas. The throughput and radiated efficiency of 


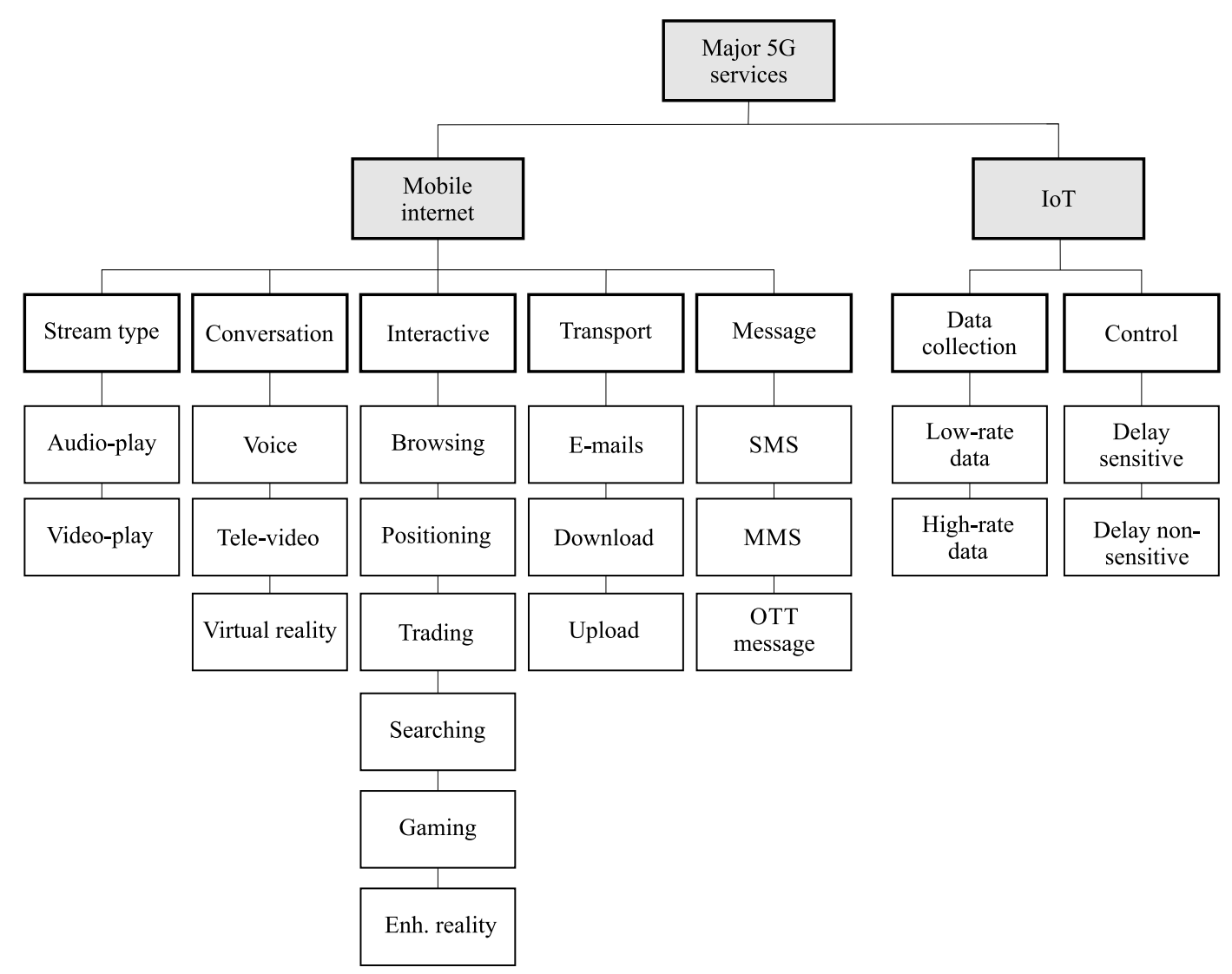

Fig. 1. Major $5 \mathrm{G}$ technology services.

the antenna can be improved by placing additional antennas to focus energy in each smaller region. It is an enabler to future broadband networks, or IoT applications combined with clouds.

In conventional MIMO systems like LTE, the base station sends out pilot waveforms. Based on that, channel response will be estimated and transmitted back. This is not possible in massive MIMO, at least when operating in a high mobility environment, for two following reasons:

1. The optimal downlink pilot should be mutually orthogonal between the antennas.

2. The number of channel responses each terminal must estimate should be proportional to the number of base station antennas.

Some major issues that should be addressed by massive MIMO, from the research point of view, are:

- fast and distributed coherent signal processing related downlink issues,

- the challenge of low cost hardware,

- hardware impairments,

- internal power consumption,
- channel characteristics,

- cost of reciprocity calibration,

- pilot contamination,

- non-CSI at TX,

- new deployment sceneries.

To overcome some of those issues, involving a like large coverage area, for instance, a planar MIMO antenna is designed with a $9 \mathrm{GHz}$ bandwidth for Ka band and 5G applications (21-30 GHz). In comparison with massive MIMO, the proposed model is not superior with respect to throughput and efficiency, but its compact dimensions and suitable gain levels render it most suitable for the desired applications. The designed antenna comprises a sigma-shaped radiator and a c-shaped coupled ground plane with CPW feeding. Simulated and measured performance shows that the MIMO antenna plays a vital role in $5 \mathrm{G}$ and $\mathrm{Ka}$ band applications.

\section{Antenna Design}

Such parameters like thickness and dielectric constant of the substrate affect impedance bandwidth of the antenna. 
(a)

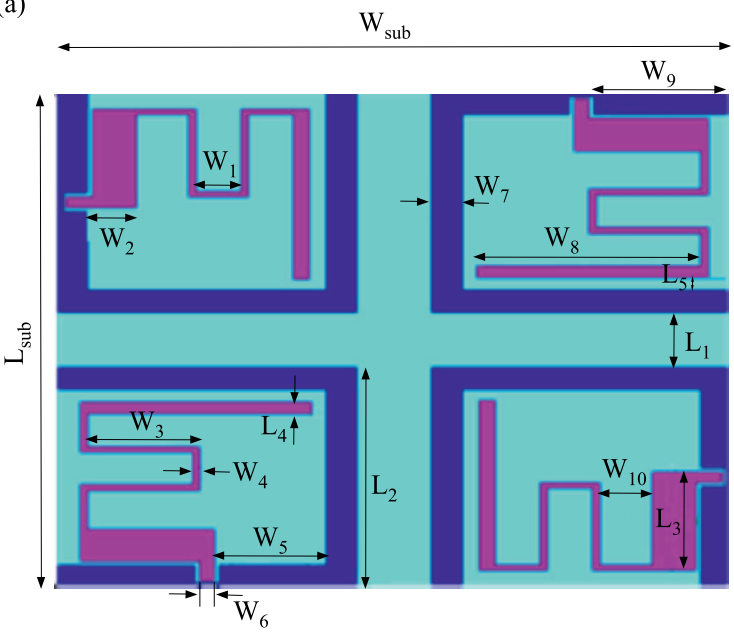

(b)

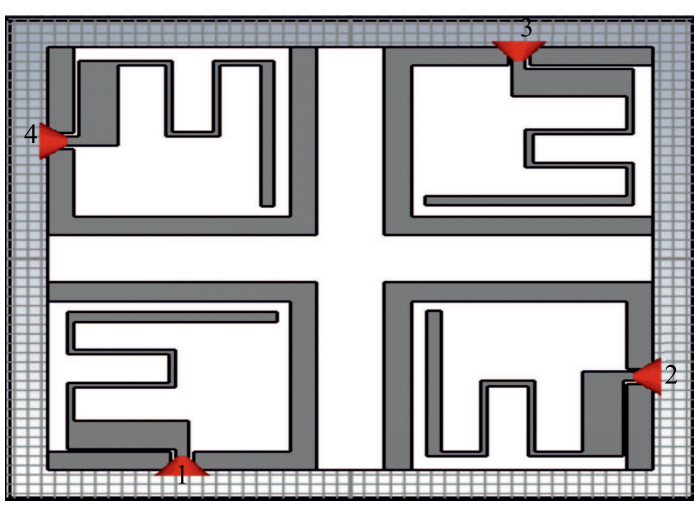

Fig. 2. MIMO antenna geometry: (a) HFSSS model, and (b) CST model.

(a)

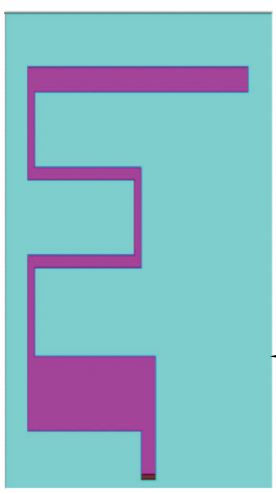

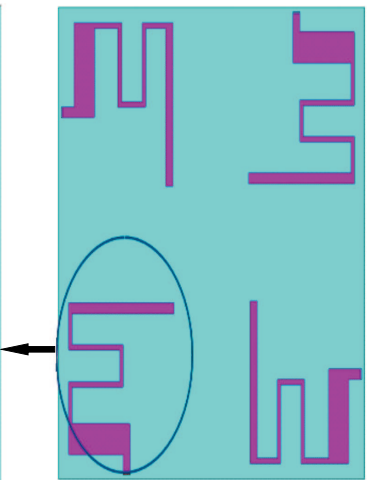

(b)

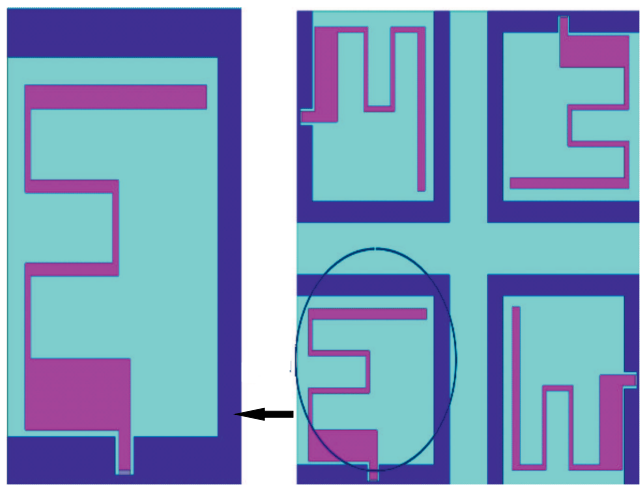

Fig. 3. MIMO antenna with: (a) patch part, and (b) patch with ground.

By increasing the height of the substrate or decreasing its permittivity, the bandwidth can be widened. The resonant frequency and impedance bandwidth will also be affected by the permittivity. Antennas manufactured on thin substrates generate more copper losses, while thicker a permittivity substrate leads to performance degradation. The configuration of the designed MIMO antenna is shown in Fig. 2. The size of the antenna proposed is $\mathrm{L}_{\text {sub }} \times \mathrm{W}_{\text {sub }}=45 \times 45 \mathrm{~mm}$. A typical FR4 laminate with $\varepsilon_{r}=4.3$ and $\tan \delta=0.02$ is used. The design is composed of two parts: a sigma-shaped monopole radiator with coplanar waveguide feeding and a c-shaped ground plane. CPW feeding is used to achieve the $50 \Omega$ impedance. When compared to microstrip line, CPW feeding has many advantages, such as easy fabrication and reduced radiation losses.

The antenna consists of four planar monopole elements with a CPW feeder through ports 1-4 respectively. The four emitters are placed perpendicularly to provide good isolation between four input ports. All sigma-shaped radiators have the same dimensions with length $\mathrm{L}_{2}$. Each of the radiators is fed by a $50 \Omega \mathrm{CPW}$ line with its width equaling W6 (Fig. 3). Table 1 shows the dimensions of the design.

The design procedure starts by finding the dimension of the antenna feeder to get $Z_{0}=50 \Omega$ using the following equations [20]:

$$
\begin{gathered}
Z_{0}=\frac{30 \pi K^{1}(k)}{\sqrt{\varepsilon_{e}} K(k)}, \\
k=\frac{s}{s+2 w_{c}}, \\
\varepsilon_{e}=\frac{\varepsilon_{r}+1}{2}(A+B), \\
A=\tanh \left(1.785 \log \frac{h}{\omega_{c}}+1.75\right), \\
B=k \frac{\omega_{c}}{h}\left[0.04-0.7 k+0.01\left(1-0.1 \varepsilon_{r}\right)(0.25+k)\right], \\
K^{1}(k)=k \sqrt{1-k^{2}} .
\end{gathered}
$$


In Eqs. (1)-(6) $s$ is the central conductor, $w_{c}$ is the slot width of CPW, $h$ is the substrate thickness, $\varepsilon_{r}$ is the dielectric constant of the substrate laminate.

Table 1

Dimensions of the MIMO antenna design

\begin{tabular}{|c|c|c|c|}
\hline Parameter & Value [mm] & Parameter & Value [mm] \\
\hline \hline $\mathrm{L}_{\text {sub }}$ & 45 & w3 & 8 \\
\hline L1 & 5 & w4 & 0.5 \\
\hline L2 & 20 & w5 & 9.2 \\
\hline L3 & 9 & w6 & 1.3 \\
\hline L4 & 1 & w7 & 2 \\
\hline L5 & 2.3 & w8 & 15.5 \\
\hline $\mathrm{W}_{\text {sub }}$ & 45 & w9 & 9.2 \\
\hline w1 & 3 & w10 & 3.5 \\
\hline w2 & 3 & - & - \\
\hline
\end{tabular}

The designed MIMO antenna was analyzed and simulated using FEM-based HFSS software and CST Microwave Studio software. The comparative analysis performed with the use of both tools, as well as its results are presented in the next section.

\section{Results and Discussion}

This section presents HFSS. The antenna parameter $\mathrm{S}_{11}$ has a significant importance, which defines the impedance matching characteristics and the bandwidth. The MIMO antenna covers bandwidth of around $9 \mathrm{GHz}(21-30 \mathrm{GHz})$

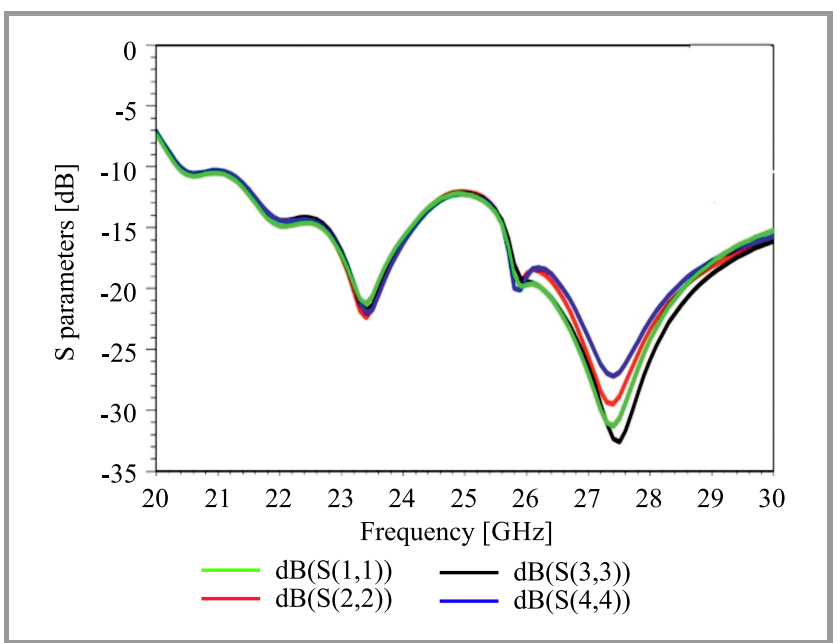

Fig. 4. Simulated reflection coefficient $\mathrm{S}_{11}$ of MIMO antenna. (For color pictures visit www.nit.eu/publications/journal-jtit)

with a return loss below $-10 \mathrm{~dB}$. The bandwidth of the antenna is satisfactory, as it covers the Ka band and 5G applications.
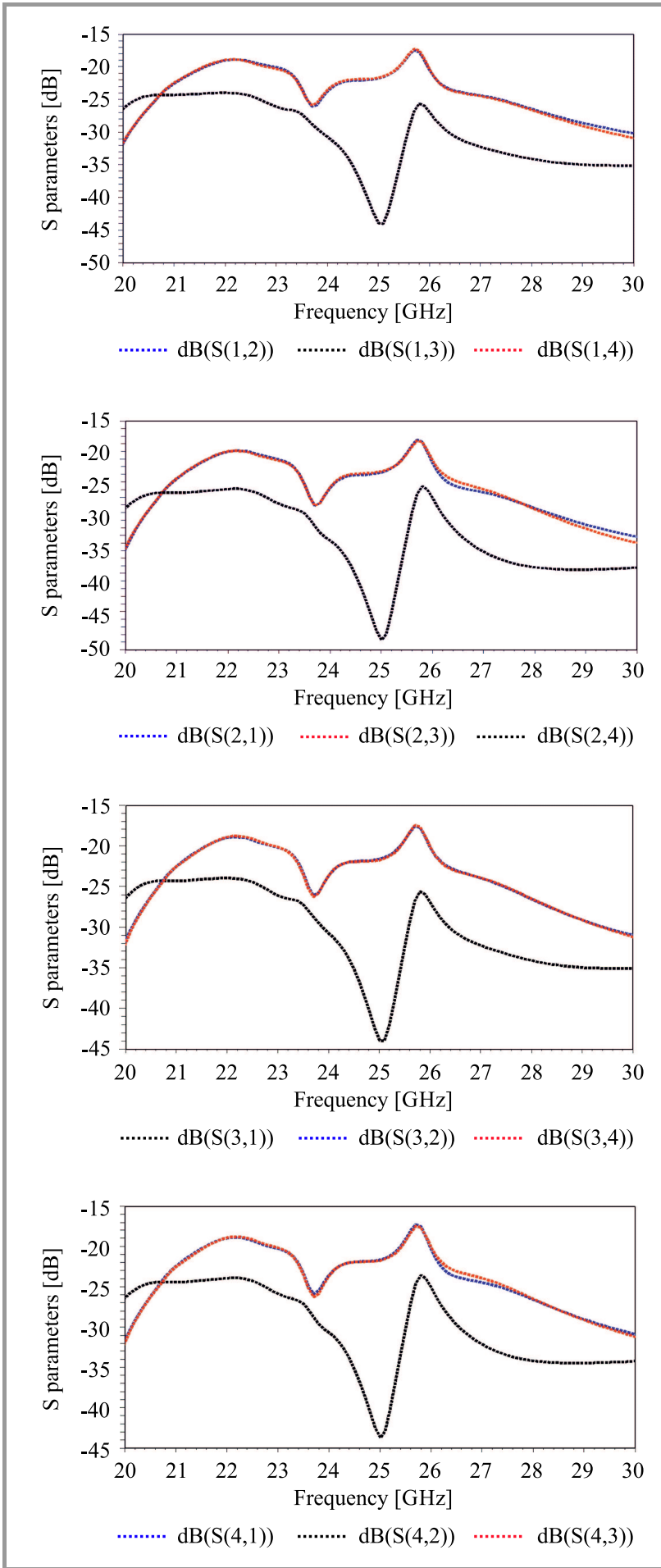

Fig. 5. Transmission coefficient result of MIMO.

The design criteria meet the satisfactory antenna return loss and bandwidth for the desired applications. The MIMO antenna has $(1 \times 4)$ basic elements, with the dimensions of $45 \times 45 \mathrm{~mm}$. Isolation is provided between each pair of basic elements to prevent coupling caused by transitions. The reflection and mutual coupling coefficients are is depicted in Fig. 4. The transmission coefficient values are below $-15 \mathrm{~dB}$, giving good significance of low mutual coupling between the elements (Fig. 5). 
(a)

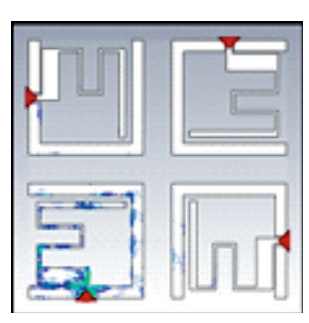

(b)

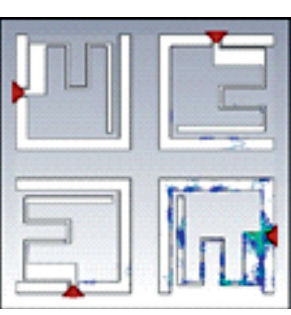

(c)

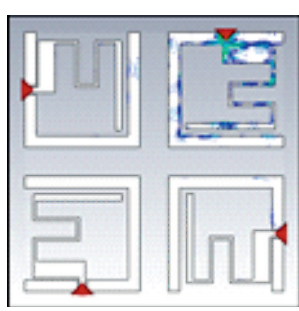

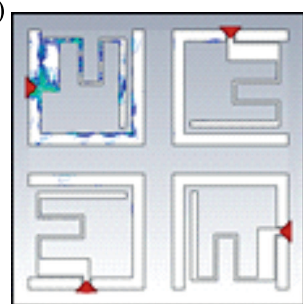

Fig. 6. Surface current distribution of a MIMO antenna at $23.5 \mathrm{GHz}$ : (a) port 1 excited and other ports terminated, (b) port 2 excited, (c) port 3 excited, and (d) port 4 excited.

(a)

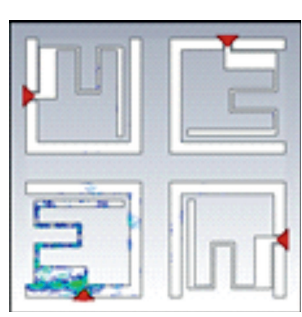

(b)

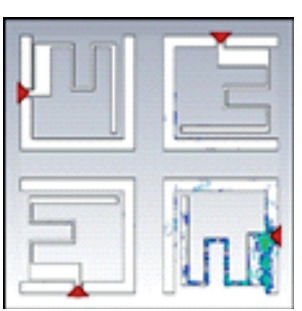

(c)

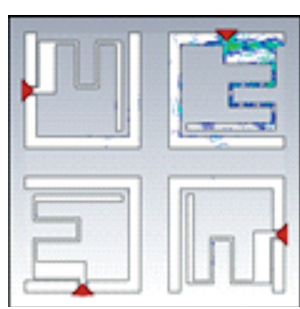

(d)

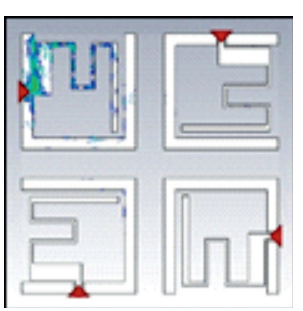

Fig. 7. Surface current distribution at $28 \mathrm{GHz}$ : (a) port 1 excited and remaining ports terminated, (b) port 2 excited, (c) port 3 excited, and (d) port 4 excited.

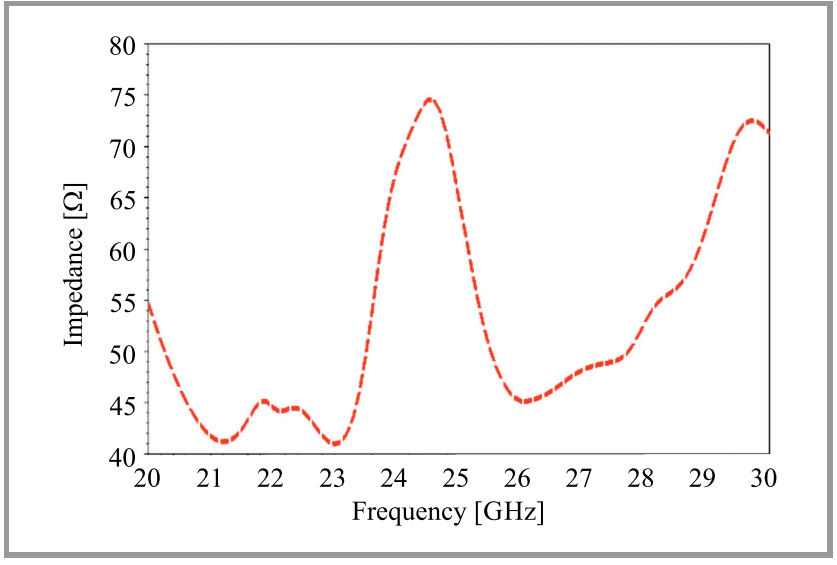

Fig. 8. Impedance response of the proposed MIMO antenna.
The reflection coefficients show that the elements of the proposed MIMO antenna have the average impedance bandwidth of $40 \%$, with the reflection coefficient of less than $-10 \mathrm{~dB}$ in $21-30 \mathrm{GHz}$. The transmission coefficients from ports 1-4 are shown in Fig. 5. Mutual coupling values are less than $-15 \mathrm{~dB}$ over the entire band.

The current distributions at resonant frequencies of 23.5 and $28 \mathrm{GHz}$ are depicted in Figs. 7 and 8. The current density on the radiating structure shows a low level of mutual coupling. When port 1 is excited and other ports are terminated, the current density is more focused on the first antenna element and very low coupling can be observed in Fig. 6a. Similarly, for other port excitations the current density on the excited port related to the radiating element and low coupling on other elements may be observed. (a)

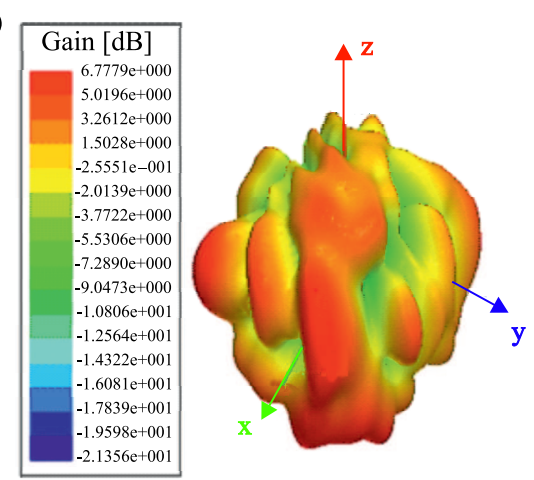

(b)

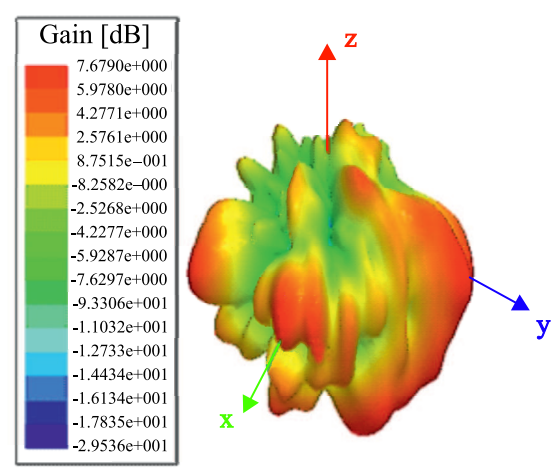

Fig. 9. 3D gain pattern with port 1 excited and remaining ports terminated at: (a) $23.5 \mathrm{GHz}$, and (b) $28 \mathrm{GHz}$. 
(a)

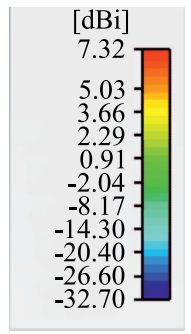

(b)

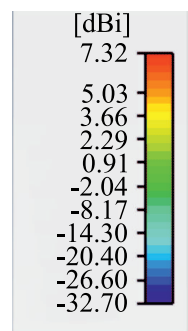

(c)

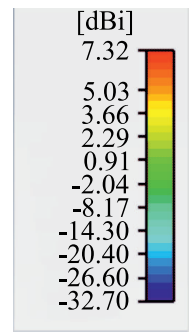

(d)

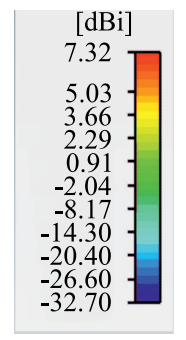

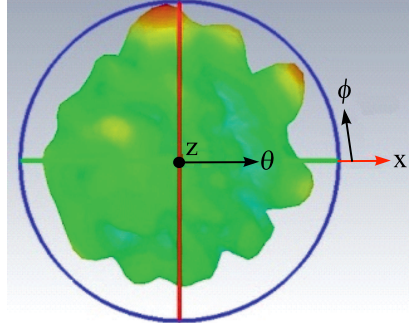
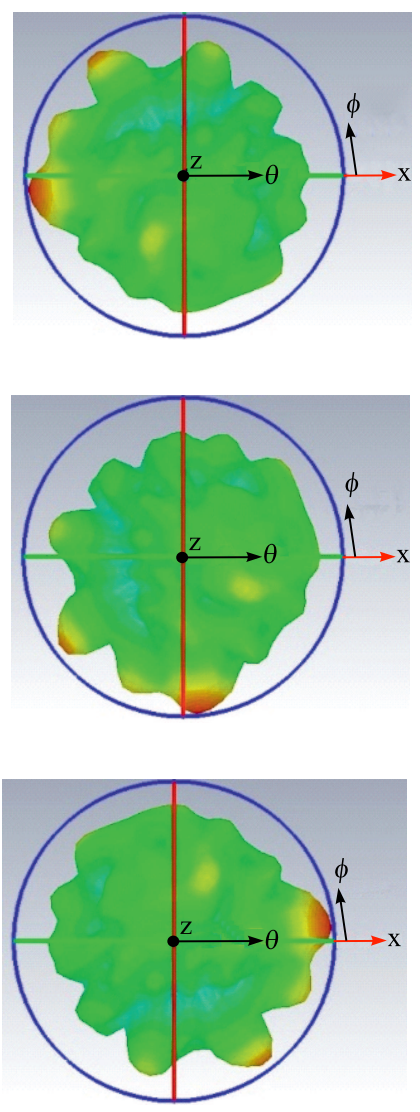
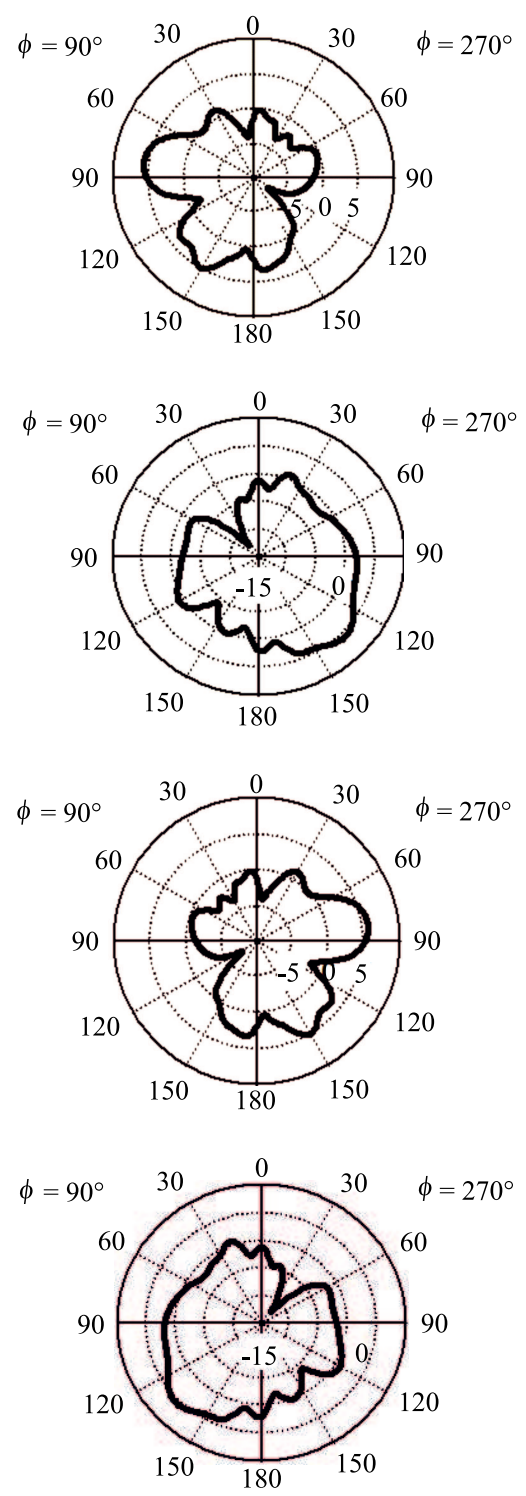

Fig. 10. Radiation pattern in $3 \mathrm{D}$ and polar representation at $23.5 \mathrm{GHz}$ : (a) port 1 excited and remaining ports terminated, (b) port 2 excited, (c) port 3 excited, and (d) port 4 excited.

The aerial impedance at resonant frequencies is depicted in Fig. 8. The MIMO antenna shows a perfect impedance of $50 \Omega$ at 23.5 and $28 \mathrm{GHz}$.

The MIMO antenna provides gain of over $7.6 \mathrm{~dB}$ within the operating range and gives minimum losses with the average efficiency of $78 \%$ over the entire band. At 23.5 and $28 \mathrm{GHz}$, the average efficiency is almost $80 \%$. The 3D gain pattern obtained with the use of the HFSS tool is shown in Fig. 9.

To show polarization diversity performance, radiation patterns for differential ports $1-4$ at 23.5 and $28 \mathrm{GHz}$ are presented in Figs. 10 and 11. These patterns are almost identical with $90^{\circ}$ rotations, which confirms the polarization diversity problem.

Figure 12 shows the frequency vs. gain and efficiency plot. The obtained gain peak is $7.3 \mathrm{~dB}$ at $23.5 \mathrm{GHz}$ and $7.5 \mathrm{~dB}$ at $28 \mathrm{GHz}$, while the average efficiency of more than $76 \%$ is achieved.

\subsection{Envelope Correlation Coefficient}

The envelope correlation coefficient (ECC) describes diversity-related performance. An ECC of $=0.5$ is generally recognized as acceptable in various conditions. The proposed aerial provides ECC $<0.03$ over its operating frequency band.

\section{Parametric Analysis}

The aerial dimensions were optimized using a parametric analysis. First, the antenna performance over the w2 parameter examined (Fig. 14). With w2 of $2 \mathrm{~mm}$ the an- 
(a)

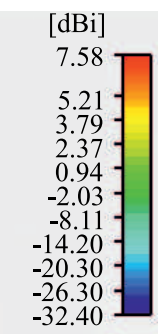

(b)

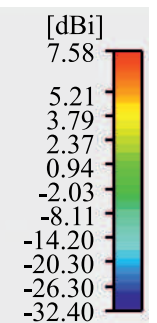

(c)

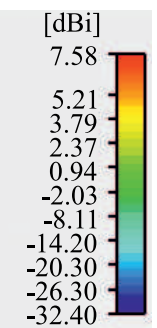

(d)

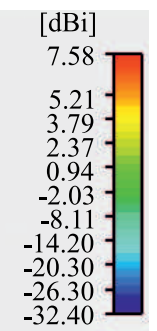

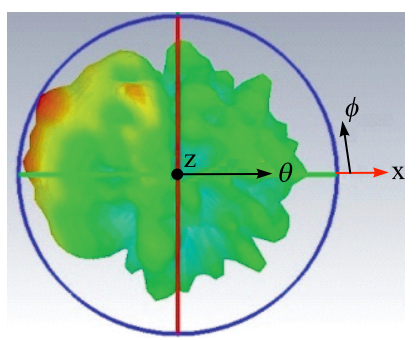
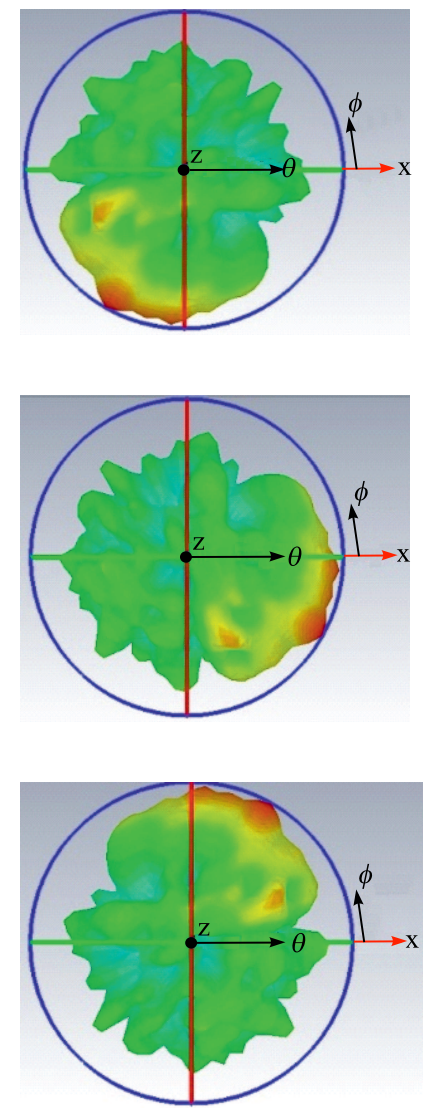
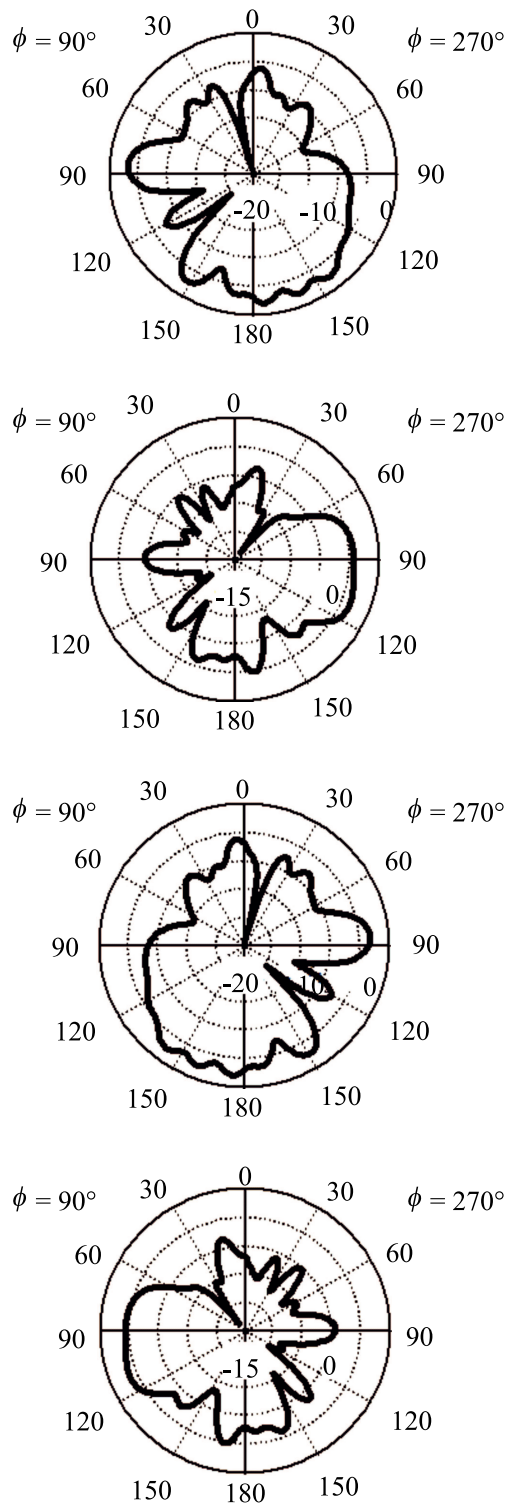

Fig. 11. Radiation pattern in 3D and polar representation at $28 \mathrm{GHz}$ : (a) port 1 excited and remaining ports terminated, (b) port 2 excited, (c) port 3 excited, and (d) port 4 excited.

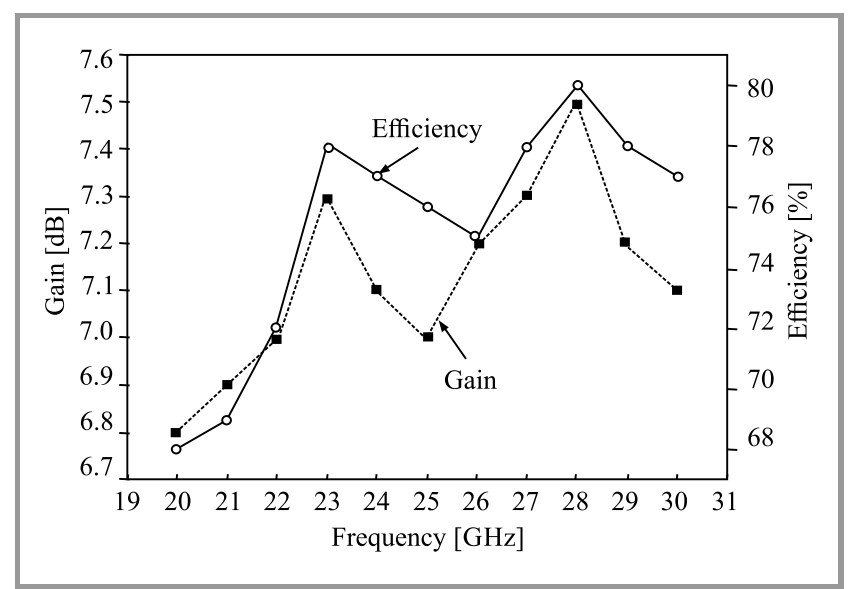

Fig. 12. Radiation efficiency and gain.

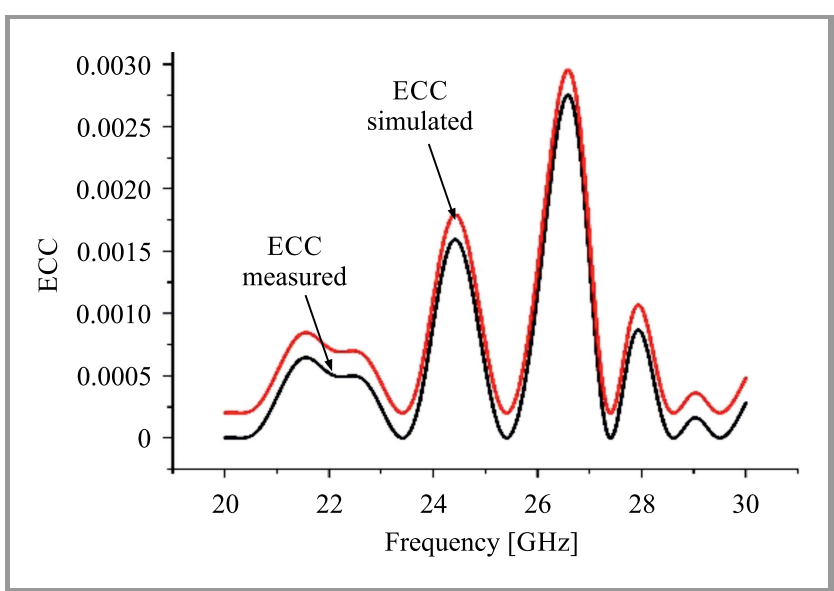

Fig. 13. Envelope correlation coefficient. 


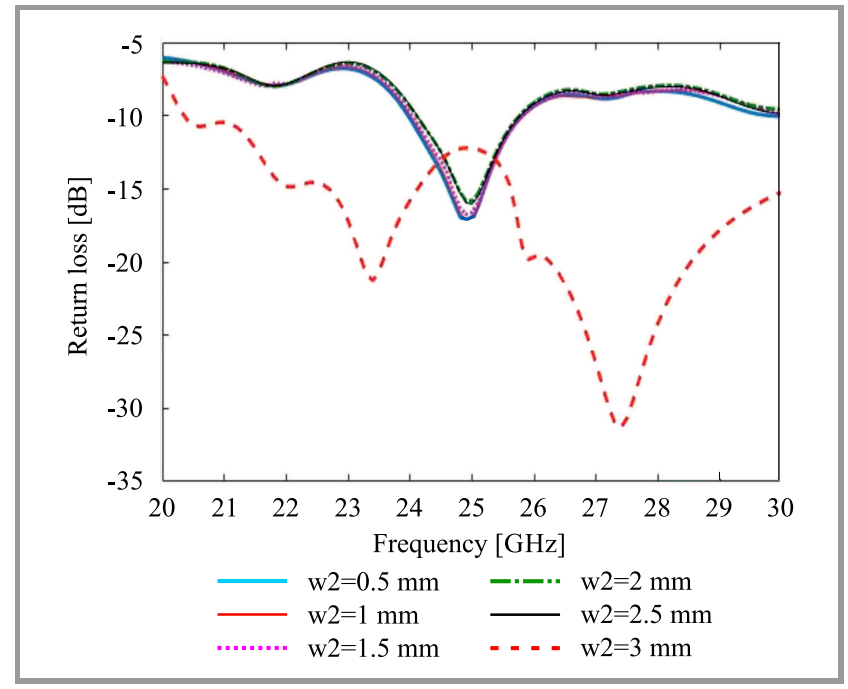

Fig. 14. Parametric analysis with change in w2.

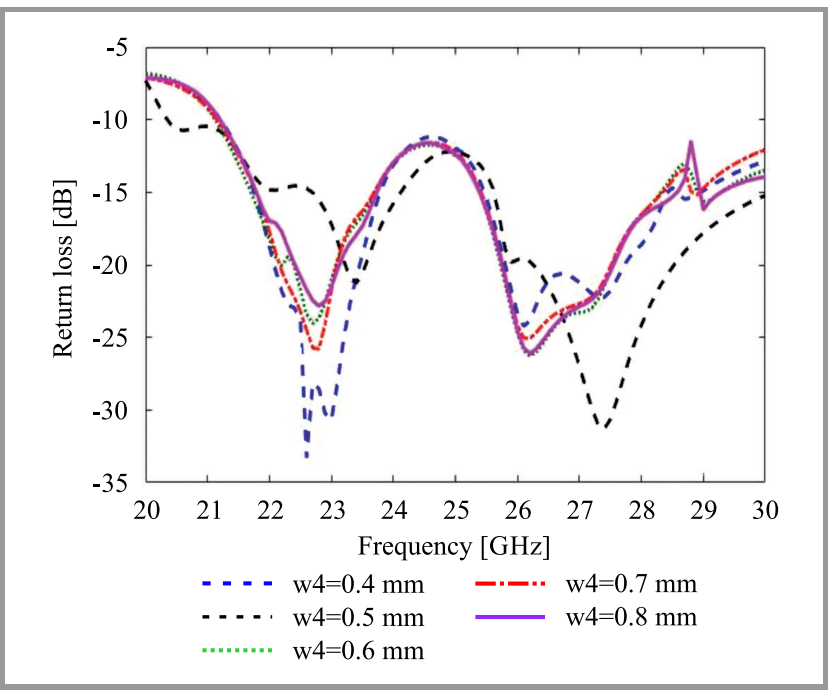

Fig. 15. Parametric analysis with change in w4.

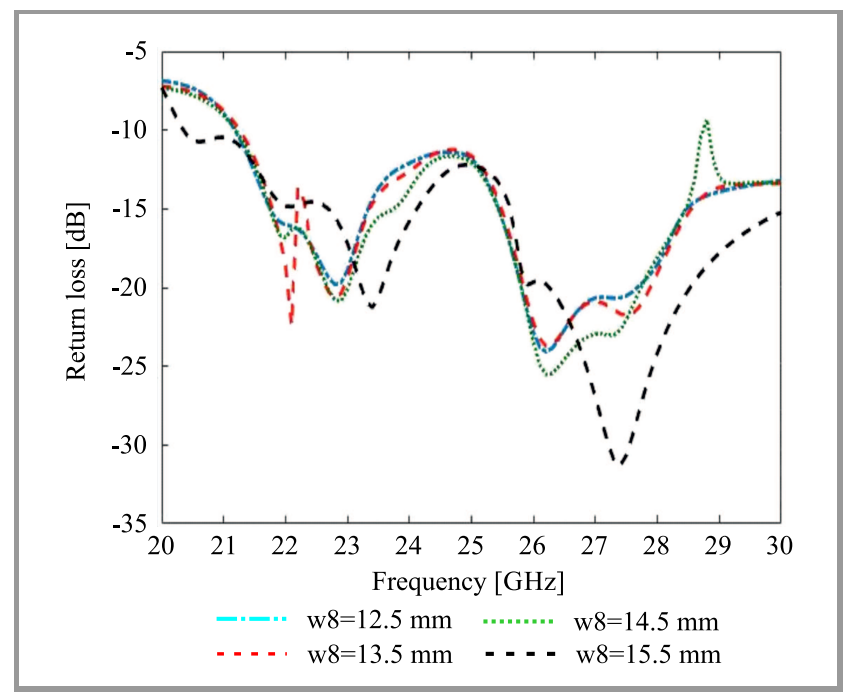

Fig. 16. Parametric analysis with change in w8.

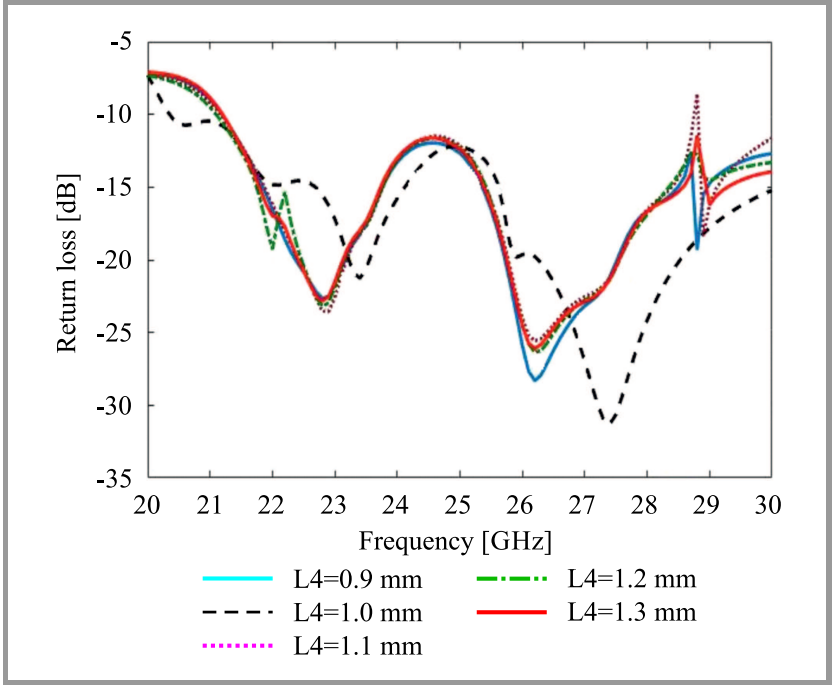

Fig. 17. Parametric analysis with change in L4.

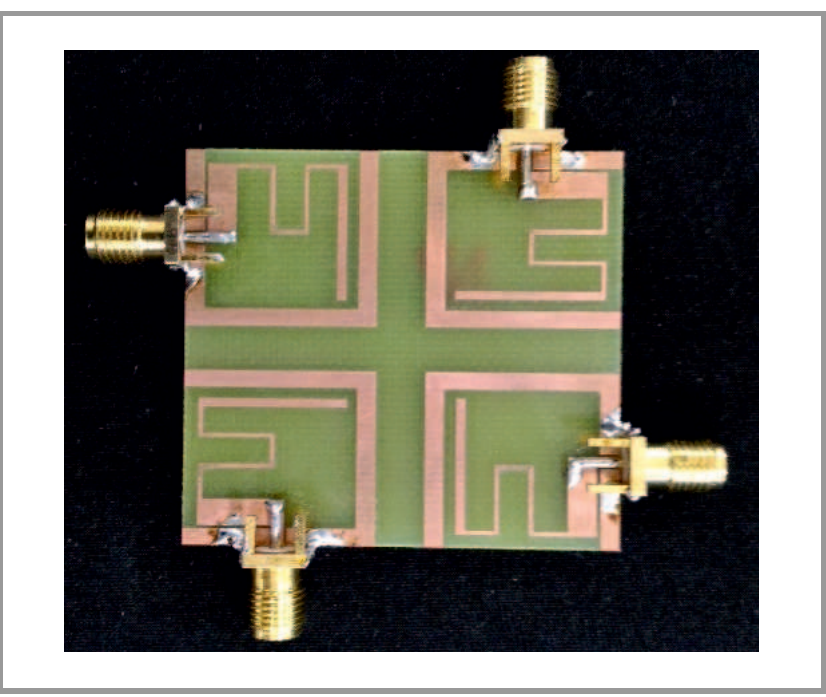

Fig. 18. MIMO antenna prototype.

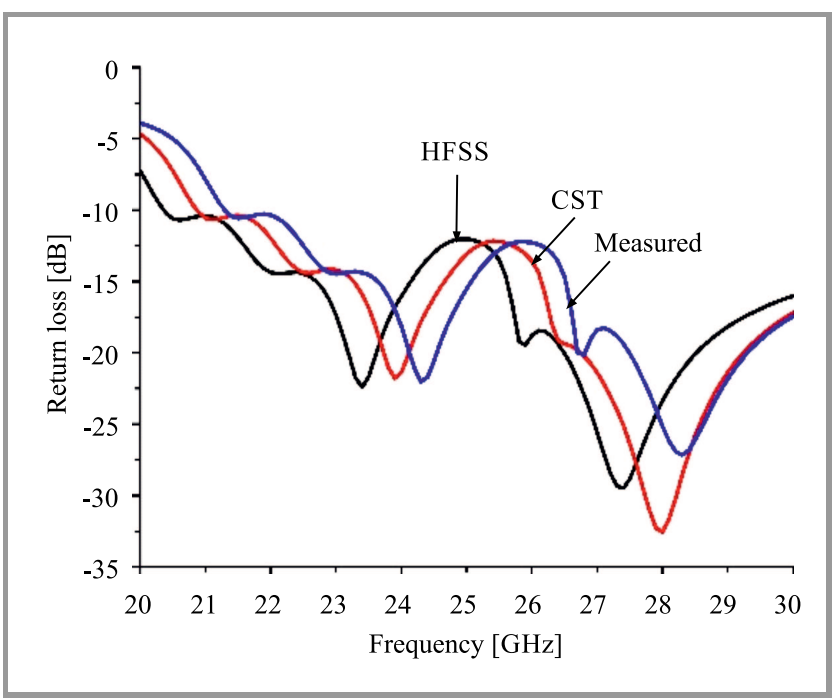

Fig. 19. Measured and simulated reflection coefficient parameter. 
tenna shows superior results. Next, w4 was varied between $0.4-0.8 \mathrm{~mm}$, and optimum performance was obtained at $0.5 \mathrm{~mm}$ (Fig. 15).

The width of radiating element's upper strip w8 was also varied from 12.5 to $15.5 \mathrm{~mm}$. The optimum performance was obtained at $15.5 \mathrm{~mm}$ (Fig. 16). For w $8=14.5 \mathrm{~mm}$, the antenna shows a notch band from 28.5 to $29.5 \mathrm{GHz}$.

Finally, the length of the radiating element's upper strip was varied from 0.9 to $1.3 \mathrm{~mm}$. For $\mathrm{L} 4=1 \mathrm{~mm}$ the antenna was showing the best impedance bandwidth characteristics (Fig. 17).

The proposed MIMO antenna was prototyped on the FR4 substrate (Fig. 18), using SMA connectors soldered to all ports. The measured $S_{11}$ reflection coefficient is compared with simulation results obtained from HFSS and CST (Fig. 19).

Table 2

Comparison of the designed MIMO antenna with other research

\begin{tabular}{|c|c|c|c|c|}
\hline Reference & $\begin{array}{c}\text { Antenna } \\
\text { dimensions } \\
{[\mathrm{mm}]}\end{array}$ & $\begin{array}{c}\text { Peak } \\
\text { gain } \\
{[\mathrm{dB}]}\end{array}$ & $\begin{array}{c}\text { Average } \\
\text { effi- } \\
\text { ciency } \\
{[\%]}\end{array}$ & Band \\
\hline \hline$[1]$ & $108 \times 135 \times 0.6$ & 7.2 & 70 & $5 \mathrm{G}$ \\
\hline$[14]$ & $136 \times 68 \times 1.6$ & 5 & 75 & $5 \mathrm{G}$ \\
\hline$[15]$ & $140 \times 76 \times 0.8$ & 3 & 45 & $5 \mathrm{G}$ \\
\hline$[16]$ & $130 \times 170 \times 1.6$ & 6.5 & 85 & $5 \mathrm{G}$ \\
\hline$[19]$ & $72 \times 17.2 \times 1.6$ & 7 & 68 & $\mathrm{Ka}$ \\
\hline Proposed & $45 \times 45 \times 1.6$ & 7.5 & 78 & $\begin{array}{c}\text { Ka } \\
\text { and } \\
5 \mathrm{G}\end{array}$ \\
\hline
\end{tabular}

Table 2 shows a comparison between the proposed model and other antennas proposed by researchers.

\section{Conclusion}

The proposed antenna model provides excellent performance characteristics within the $9 \mathrm{GHz}$ bandwidth, has a gain peak of $7.5 \mathrm{~dB}$ and achieves good polarization. Even though it is not superior to massive MIMO with respect to high-speed broadband network and advanced IoT applications, its compactness, low diversity and adaptability to advanced communication modules makes this antenna model more suitable for Ka band and 5G applications.

\section{Acknowledgements}

We like to thank the ECE department of KLU and DST for the technical support through ECR/2016/000569 and EEQ /2016/000604.

\section{References}

[1] A. Dadgarpour, M. S. Sorkherizi, and A. A. Kishk, "High efficient circularly polarized magneto-electric dipole antenna for 5G applications using dual-polarized split-ring resonator lens", IEEE Transact. on Antenn. and Propag., vol. 65, no. 8, pp. 4263-4267, 2017 (doi: 10.1109/TAP.2017.2708091).

[2] Y. Yao, X. Chung, Ch. Wang, J. Yu, and X. Chen, "Wideband circularly polarized antipodal curvedly tapered slot antenna array for 5G applications", IEEE J. on Select. Areas in Commun., vol. 35, no. 7, pp. 1539-1549, 2017 (doi: 10.1109/JSAC.2017.2699101).

[3] W. Hong et al., "Multi-beam antenna technologies for 5G wireless communications", IEEE Transact. on Antenn. and Propag., vol. 99, no. 12 , pp. $6231-6249,2017$

(doi: 10.1109/TAP.2017.2712819).

[4] A. Khuran and S. R. Rosenthal, "Integrating the fuzzy front end of new product development”, MIT Sloan Manag. Rev., vol. 38, no. 4, pp. 103-120, 1997.

[5] Ch. Zhang, Y.-H. Huang, F. Sheikh and Zh. Wang, "Advanced baseband processing algorithms, circuits, and implementations for 5G communication", IEEE J. on Emerging and Select. Topics in Circ. and Sys., vol. 7, no. 4, pp. 477-490, 2017

(doi: 10.1109/JETCAS.2017.2743107).

[6] V. Jungnickel et al., "The role of small cells, coordinated multipoint, and massive MIMO in 5G", IEEE Commun. Mag., vol. 52, no. 5, pp. 44-51, 2014 (doi: 10.1109/MCOM.2014.6815892).

[7] Y. Yifei and Zh. Longming, "Application scenarios and enabling technologies of 5G", China Commun., vol. 11, no. 11, pp. 69-79, 2014 (doi: 10.1109/CC.2014.7004525).

[8] R. Glogowski, J.-F. Zurcher, C. Peixeiro, and J. R. Mosig, "A lowloss planar Ka-band antenna subarray for space applications", IEEE Transact. on Antenn. and Propag., vol. 61, no. 9, pp. 4549-4557, 2013 (doi: 10.1109/TAP.2013.2264452).

[9] Luo, Qi, Steven Gao, Chong Zhang, Dawei Zhou, Tobias Chaloun, Wolfgang Menzel, Volker Ziegler, and Mohammed Sobhy, "Design and analysis of a reflectarray using slot antenna elements for Kaband SatCom", IEEE Transact. on Antenn. and Propag., vol. 3, no. 4, pp. 1365-1374, 2015 (doi: 10.1109/TAP.2015.2401393).

[10] Y. Ch. Lee and Ch. S. Park, "A compact broadband PHEMT MMIC power amplifier for K through Ka-band applications", AEU-Int. J. of Electr. and Commun., vol. 57, no. 4, pp. 291-294, 2003 (doi: 10.1078/1434-8411-54100174).

[11] M. R. Chaharmir and J. Shaker, "Design of a multilayer X-/Kaband frequency-selective surface-backed reflectarray for satellite applications", IEEE Transact. on Antenn. and Propag., vol. 63, no. 4, pp. 1255-1262, 2015 (doi: 10.1109/TAP.2015.2389838).

[12] B. Rohrdantz et. al, "An electronically scannable reflector antenna using a planar active array feed at Ka-band", IEEE Transact. on Microwave Theory and Techniq., vol. 65, no. 5, pp. 1650-1661, 2017 (doi: 10.1109/TMTT.2017.2663402).

[13] D. S. Ramkiran et al., "Coplanar wave guide fed dual band notched MIMO antenna", Int. J. of Electr. and Computer Engineer., vol. 6, no. 4, pp. 1732-1741, 2016 (doi: 10.11591/ijece.v6i4.1057).

[14] M.-Y. Li et al., "Eight-port orthogonally dual-polarized antenna array for 5G smartphone applications", IEEE Transact. on Antenn. and Propag., vol. 64, no. 9, pp. 3820-3830, 2016

(doi: 10.1109/TAP.2016.2583501).

[15] M. S. Sharawi, M. Ikram, and A. Shamim, "A two concentric slot loop based connected array MIMO antenna system for $4 \mathrm{G} / 5 \mathrm{G}$ terminals", IEEE Transact. on Antenn. and Propag., vol. 65, no. 12 pp. 6679-6686, 2017 (doi: 10.1109/TAP.2017.2671028).

[16] Y.-L. Ban, Ch. Li, Ch.-Y.-D. Sim, G. Wu, and K.-L. Wong, "4G/5G multiple antennas for future multi-mode smartphone applications", IEEE Access, vol. 4, pp. 2981-2988, 2016 (doi: 10.1109/ACCESS.2016.2582786).

[17] J. A. Perez García, S. Kosmopoulos, and G. Goussetis, "A compact 12-way slotted waveguide power combiner for Ka-band applications", IEEE Microwave and Wirel. Compon. Let., vol. 27, no. 2, pp. 135-137, 2017 (doi: 10.1109/LMWC.2016.2646903). 
[18] M. V. Komandla, Gh. Mishra, and S. K. Sharma, "Investigations on dual slant polarized cavity-backed massive MIMO antenna panel with beamforming", IEEE Transact. on Antenn. and Propag., vol. 65, no. 12, pp. 6794-6799, 2017 (doi: 10.1109/TAP.2017.2748239).

[19] M. Lin, P. Liu, and Zh. Guo, "Gain-enhanced Ka-band MIMO antennas based on the SIW corrugated technique", IEEE Antenn. and Wirel. Propag. Let., vol. 16, pp. 3084-3087, 2017 (doi: 10.1109/LAWP.2017.2761903).

[20] J. Ch. Rao et al., "Compact UWB MIMO slot antenna with defected ground structure", ARPN J. of Engineer. and Applied Sciences, vol. 11, no. 17, pp. 10487-10495, 2016.

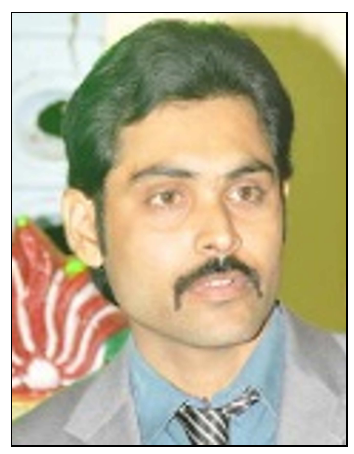

B. T. P. Madhav received his B.Sc., M.Sc., MBA, M.Tech. degrees from Nagarjuna University, Andhra Pradesh, India in 2001, 2003, 2007, and 2009 respectively. He obtained his $\mathrm{Ph} . \mathrm{D}$. in the field of antennas from Konenu Lakshaiah University. Currently he is working as a Professor and Communication Systems research Head at the Electronics and Communication Engineering Department. His research interests include antennas, liquid crystal applications and wireless communications.

E-mail: btpmadhav@kluniversity.in

Department of Electronics \& Communication Engineering Koneru Lakshmaiah Education Foundation Andhra Pradesh, India

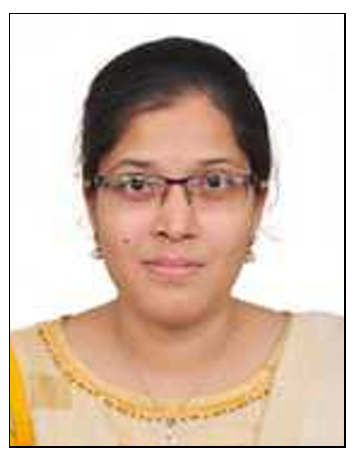

G. Jyothsna Devi is pursuing her master's degree at the Department of Electronics \& Communication Engineering of Konenu Lakshmaiah University. Her area of interest includes MIMO antennas for wireless applications, massive MIMO, compact and low profile antennas.
E-mail: jyothsnagudiseva@gmail.com

Department of Electronics \& Communication Engineering Koneru Lakshmaiah Education Foundation

Andhra Pradesh, India

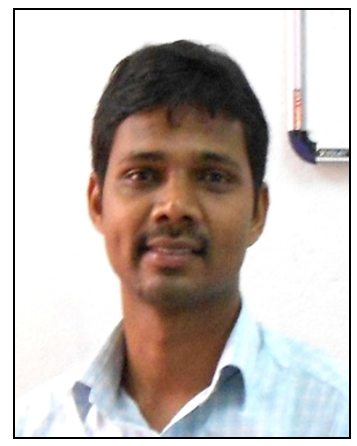

Papula Lakshman received his M.Sc. degree in electronics and communication from Andhra University, Visakhapatnam, India, and his M.Tech. degree in Microwave Engineering from GITAM University, Visakhapatnam, India. He received his Ph.D. degree from IIT Bhubaneswar, Odisha, India. His research interests include antennas, array antennas, optimization methods, EMI/EMC and soft computing.

E-mail: lakshmanpappula@kluniversity.in

Department of Electronics \& Communication Engineering Koneru Lakshmaiah Education Foundation

Andhra Pradesh, India

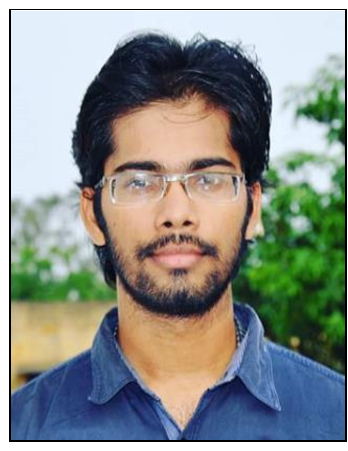

T. Anilkumar received his B.Tech. degree in Electronics and Communication Engineering from Acharya Nagarjuna University in 2014 and M.Tech. degree in Communication Systems from Andhra University in 2016. He is currently pursuing his Ph.D. at Antennas and Liquid Crystals Research Center of ECE Department, Konenu Lakshaiah University. His current research interests include reconfigurable, tunable microstrip antennas, conformal antennas and liquid crystal polymer-based antennas for vehicular communication.

E-mail: t.anilkumar@kluniversity.in

Department of Electronics \& Communication Engineering Koneru Lakshmaiah Education Foundation Andhra Pradesh, India 